\title{
Development of Novel Magnetic Responsive Intelligent Fluid, Hybrid Fluid (HF), for Production of Soft and Tactile Rubber
}

\author{
Kunio Shimada ${ }^{1}$, Ryo Ikeda ${ }^{2}$, Hiroshige Kikura², Hideharu Takahashi² \\ ${ }^{1}$ Department of Symbiotic Systems Sciences, Fukushima University, Fukushima, Japan \\ ${ }^{2}$ Research Laboratory for Nuclear Reactors, Tokyo Institute of Technology, Tokyo, Japan \\ Email: ^shimadakun@sss.fukushima-u.ac.jp, ikeda.r.ah@m.titech.ac.jp, \\ kikura@lane.iir.titech.ac.jp, htakahashi@lane.iir.titech.ac.jp
}

How to cite this paper: Shimada, K., Ikeda, R., Kikura, H. and Takahashi, H. (2021) Development of Novel Magnetic Responsive Intelligent Fluid, Hybrid Fluid (HF), for Production of Soft and Tactile Rubber. World Journal of Mechanics, 11, 187-203. https://doi.org/10.4236/wjm.2021.1110014

Received: October 11, 2021

Accepted: October 28, 2021

Published: October 31, 2021

Copyright $\odot 2021$ by author(s) and Scientific Research Publishing Inc. This work is licensed under the Creative Commons Attribution International License (CC BY 4.0).

http://creativecommons.org/licenses/by/4.0/

(c) (i) Open Access

\begin{abstract}
For the purpose of the replacement of Magnetic Fluid (MF) which is effective in the production of an artificial soft and tactile skin for the robot, etc. by utilizing a rubber solidification method with electrolytic polymerization, we proposed a novel magnetic responsive intelligent fluid, Hybrid Fluid (HF). HF is structured with water, kerosene, silicon oil having Polydimethylsiloxane (PDMS) and Polyvinyl Alcohol (PVA) as well as magnetic particles and surfactant. The state of HF changes as jelly or fluid by their rates of the constituents and motion style. In the present paper, we presented the characteristics of HF: the viscosity and the magnetization are respectively equivalent to those of other magnetic responsive fluids, MF and their solvents. For the structure, HF is soluble simultaneously with both diene and non-diene rubbers. The diene rubber such as Natural Rubber (NR) or Chloroprene (CR) has a role in the feasibility of electrolytic polymerization and the non-diene rubber such as silicon oil rubber (Q) has a role in defense against deterioration. Therefore, the electrolytically polymerized HF rubber by mixing NR, CR as well as Q is effective for the artificial soft and tactile skin. It is responsive to pressure and has optimal property on piezoelectricity in the case of the mixture of Ni particles as filler. HF is effective in the production of the artificial soft and tactile skin made of rubber.
\end{abstract}

\section{Keywords}

Intelligent Fluid, Hybrid Fluid (HF), Magnetic Fluid, Magnetic Compound Fluid (MCF), Piezoelectric Effect, Rubber, Artificial Skin, Sensor, Electrolytic Polymerization, Magnetic Cluster, Magnetic Field, Artificial Skin, Robot 


\section{Introduction}

Intrinsically a robot needs to have the soft and tactile skin for the activity in our daily life or variegated industrial places which meets intimacy with humans, for example, nursery by nursing care robot in homes, hospitals, geriatric health services facilities, etc. In order to achieve the prerequisite of the soft peculiarity for the robot skin, the utilization of rubber is shortcut because the material has elasticity and flexibility. On the other hand, the ordinary way to have the rubber tactile has been conducted on by mixing magnetic particles or carbon powder: silicon oil rubber (Q) [1] [2] [3], Chloroprene Rubber (CR) [4], Styrene-Butadiene Rubber (SBR) [5], Acrylonitrile-Butadiene Rubber (NBR) [6] and Natural Rubber (NR) [7]. The tactile peculiarity is almost piezo-element enough to be convenient on size and weight because the redundancy of essential equipment such as the external power source attached with electric wires may not be needed. However, the current soft and tactile rubber has many problems such as expensive and intricate production by drying method of the rubber vulcanization, which brings about the hardness to the rubber compared to the flexibility like the human skin.

In order to achieve the soft and tactile rubber, we previously proposed techniques for the fabrication of tactile rubber utilizing the electrolytic polymerization method [8] and Magnetic Compound Fluid (MCF) [9]. The novel solidification method is expensive and convenient production of the solidified rubber: as soon as a $6 \mathrm{~V}$ and $2.7 \mathrm{~A}$ electric field is supplied between the rubber latex sandwiched between the electrodes having $1 \mathrm{~mm}$ thickness, the rubber can be easily solidified. The rubber latex may involve any fillers and be whether diene or non-diene rubber with using surfactant or Polyvinyl Alcohol (PVA).

MCF is an intelligent colloidal fluid that is responsive to a magnetic field and is constructed by $1 \mu \mathrm{m}$ ordered metal particles such as $\mathrm{Ni}, \mathrm{Fe}, \mathrm{Cu}$, etc. and $10 \mathrm{~nm}$ ordered sphere magnetite $\left(\mathrm{Fe}_{3} \mathrm{O}_{4}\right)$ particles in a solvent such as water, kerosene, silicone oil, etc., since MCF is made by compounding metal particles and a Magnetic Fluid (MF). The application of a magnetic field then makes the metal particles and $\mathrm{Fe}_{3} \mathrm{O}_{4}$ aggregate to a magnetic cluster such a needle-like shape [10]. At first, regarding without using an electric field, when MCF is mixed into a rubber (which is then called MCF rubber) and vulcanized under a magnetic field, magnetic clusters are constructed in the rubber such that the rubber becomes responsive to a magnetic field, has magnetic and mechanical anisotropy and its conductivity enhances [11]. Next, by applying an electric field during vulcanization as well, the MCF rubber is solidified; this is a new rubber solidification method that is different from ordinary vulcanization with sulfur. The new solidification method is electrolytic polymerization in which the $\mathrm{C}=\mathrm{C}$ bonds of diene rubbers such as NR and CR are cross linked by an electric field [12]. During the electrolytic polymerization, a magnetic field is applied such that the solidified MCF rubber forms many magnetic clusters. The fabrication induces the piezo effect as well as higher conductivity [13]. In addition, the MCF rubber has higher elasticity and 
compressibility than current conductive rubber [8]. Therefore, MCF is a rubber that is both soft enough and conductive enough for haptic sensing by measuring voltage and electric current or resistivity. MCF rubber is therefore suitable as a tactile rubber.

In general, rubber is categorized into diene and non-diene rubbers. The former is prone to deterioration because of their $\mathrm{C}=\mathrm{C}$ bonds, while the latter has a certain defense against deterioration because they have C-C bonds, as seen in Ethylene-Propylene Rubber (EPM or EPDM), Urethane Rubber (U) and Q. Although the diene rubbers are water-soluble enough to be vulcanized by electrolytic polymerization, which involves the electrolysis of aqueous electrolytes, this is impossible in the case of non-diene rubbers because they do not contain water. In order to make electrolytic polymerization feasible on non-diene rubbers to take advantage of their defense against deterioration, we can mix diene and non-diene rubbers by emulsion polymerization using PVA [14] or a surfactant [15].

MCF is suitable for the production of the desired rubber with these electrolytic polymerization techniques. However, the MF that is involved in MCF is both expensive and inconvenient as it must be purchased from a company; it cannot be easily produced in the laboratory and its constituents are obscure because of a confidential engineering patent. In addition, the MF to be used in electrolytic polymerization must be water-based and it is difficult to produce enough for it to be reliably commercially available. The utilization of MF also leads that the consummated fabrication is expensive ultimately. Therefore, in the present study, we propose a cutting-edge magnetic responsive fluid, HF (hybrid fluid), that is suitable and easily producible for fabricating rubber with characteristics similar to those of human skin tissue and that can replace MCF [16]. The purpose of the present study to is the creation of new tractable responsive fluid replacing MF for the production of haptic rubber skin enough to be novel. In addition, HF is such a novel fluid that it can be soluble in both water and kerosene or silicon oil from the cause that tactile rubber to be electrolytically polymerized consists of diene and non-diene rubbers can be produced with using the HF. The characteristics of the ordinary magnetic responsive fluid such as MF, MCF and Magneto-Rheological Fluid (MRF) soluble to either water or kerosene, silicon oil has been elucidated in the field of an intelligent fluid. However, the characteristics of the intelligent fluid soluble to both water and kerosene or silicone oil have been unclear until recently. Therefore, it is novel to investigate the characteristics of HF soluble to both water and kerosene or silicon oil.

\section{Hybrid Fluid (HF)}

In order to take advantage of the defense against deterioration of non-diene rubber, diene and non-diene rubbers are combined using PVA. Diene rubbers such as NR and CR are soluble in water, while non-diene rubbers such as $\mathrm{Q}$ are soluble in kerosene. Most particularly, $\mathrm{Q}$ is soluble in silicone oil with Polydime- 
thylsiloxane (PDMS). Therefore, rubber latex combined with NR (Ulacol; Rejitex Co., Ltd., Atsugi, Japan), CR (671A; Showa Denko Co., Ltd., Tokyo, Japan) and $\mathrm{Q}$ can be soluble in water, kerosene or silicone oil. For the possible combination of rubber and magnetic responsive fluid, therefore, the magnetic responsive fluid may include water, kerosene or silicone oil. On the other hand, the magnetic responsive fluid that replaces $\mathrm{MF}$ consists of magnetic particles and surfactant, which is simulated MF whose $\mathrm{Fe}_{3} \mathrm{O}_{4}$ is coated by a surfactant. Thus, the new magnetic responsive fluid consists of $\mathrm{Fe}_{3} \mathrm{O}_{4}$ (Fujifilm Wako Chemicals Co., Ltd., Osaka, Japan) and Fe (M300, about 50- $\mu$ m particles; Kyowa Pure Chemical Co., Ltd., Tokyo, Japan) particles in the solution mixed with water, kerosene or silicone oil (KF96 with 1-cSt viscosity with PDMS; Shin-Etsu Chemical Co., Ltd., Tokyo, Japan) with a surfactant of sodium hexadecyl sulfate aqueous solution (Fujifilm Wako Chemicals), where KF96 involves PDMS. M300 should be compounded because the mixed fluid has little responsive to a magnetic field when we use only $\mathrm{Fe}_{3} \mathrm{O}_{4}$. The purpose of using the surfactant is to optimize the combination of diene and non-diene rubbers, as shown in our previous study [15]. The constituents are shown in Table 1. HF is produced as follows. First, sodium hexadecyl sulfate is combined with water at a weight ratio of $1: 10$. This sodium hexadecyl sulfate aqueous solution is compounded with $\mathrm{Fe}_{3} \mathrm{O}_{4}$ and $\mathrm{M} 300$ using an agitator. Next, the mixture is agitated during about 1 hour with water, kerosene, KF96 and PVA. The using of high-speed agitator or the mixing under vacuum optimizes well-dispersion enough to avoid the separated state of the mixed liquids. We should pay attention of degassing from the liquids since the liquids inflate by the agitating. If we don't use the vacuum pump during agitating, the liquids must be vacuumed intermittently in the interval of agitating.

Table 1. Constituents of Hybrid Fluids (HFs): PVA, polyvinyl alcohol; non-mag., HF with no application of a magnetic field; mag., with the application of a magnetic field at $180 \mathrm{mT}$; under, under the application of a magnetic field; after removing, soon after removing the magnetic field.

\begin{tabular}{|c|c|c|c|c|c|c|c|c|c|c|c|}
\hline \multirow{3}{*}{$\begin{array}{l}\text { Specimen } \\
\text { No. }\end{array}$} & \multirow{3}{*}{$\begin{array}{c}\text { Water } \\
{[\mathrm{g}]}\end{array}$} & \multirow{3}{*}{$\begin{array}{c}\text { Kerosene } \\
{[\mathrm{g}]}\end{array}$} & \multirow{3}{*}{$\begin{array}{c}\text { KF96 (1cSt) } \\
{[\mathrm{g}]}\end{array}$} & \multirow{3}{*}{ PVA [g] } & \multirow{3}{*}{$\begin{array}{c}\mathrm{Fe}_{3} \mathrm{O}_{4} \\
{[\mathrm{~g}]}\end{array}$} & \multirow{3}{*}{$\begin{array}{l}\text { M300 } \\
{[\mathrm{g}]}\end{array}$} & \multirow{3}{*}{$\begin{array}{l}\text { Sodium hexadecyl } \\
\text { sulfate aqueous } \\
\text { solution [g] }\end{array}$} & \multirow{3}{*}{$\begin{array}{l}\text { Density } \\
{\left[\mathrm{kg} / \mathrm{m}^{3}\right]}\end{array}$} & \multicolumn{3}{|c|}{ Corresponding to Figure 1} \\
\hline & & & & & & & & & & & \\
\hline & & & & & & & & & & unde & removing \\
\hline HF1 & 3 & 3 & 3 & 3 & 3 & 3 & 4 & 1230 & (a) & (b) & (c) \\
\hline HF2 & 3 & 3 & 3 & 21 & 3 & 3 & 4 & 1041 & (d) & (e) & (f) \\
\hline HF3 & 3 & 3 & 3 & 3 & 3 & 3 & 2 & 1184 & (g) & (h) & (i) \\
\hline HF4 & 3 & 2 & 2 & 1.6 & 1 & 4 & 5 & & (j) & & \\
\hline HF5 & 3 & 3 & 3 & & 3 & 3 & 4 & 1368 & $(\mathrm{k})$ & (l) & (m) \\
\hline HF6 & 3 & & 3 & 3 & 3 & 3 & 4 & 1186 & (n) & (o) & (p) \\
\hline HF7 & & 1 & 1 & 1 & 0.22 & 1 & 0.8 & & (q) & & \\
\hline HF8 & & 3 & 3 & 3 & 3 & 3 & 4 & 1320 & $(\mathrm{r})$ & (s) & $(\mathrm{t})$ \\
\hline HF9 & 3 & 3 & & 3 & 3 & 3 & 4 & 1393 & (u) & (v) & (w) \\
\hline
\end{tabular}




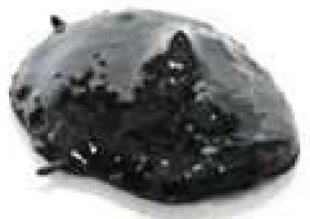

(a)

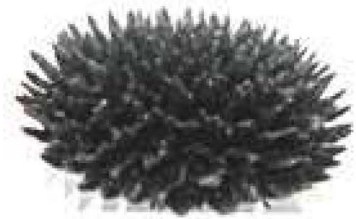

(e)

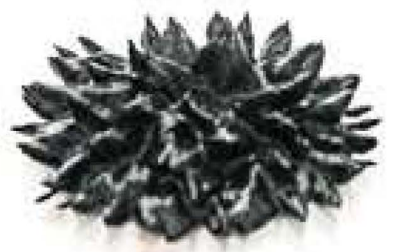

(i)

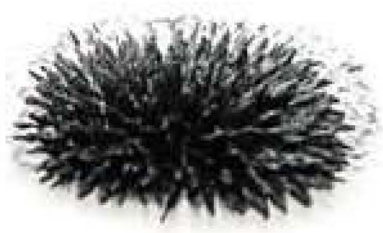

(m)

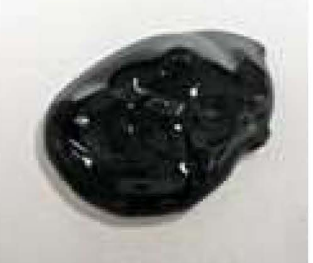

(q)

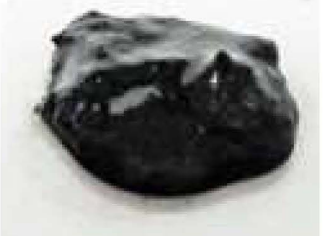

(u)

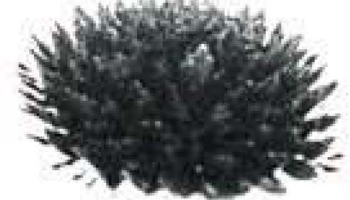

(b)

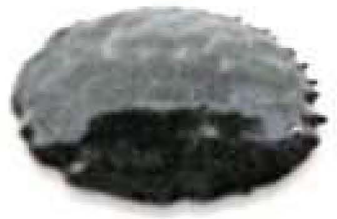

(f)

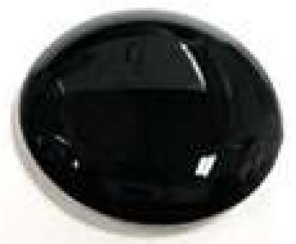

(j)

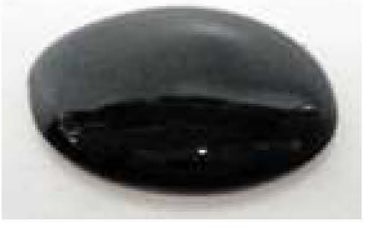

(n)

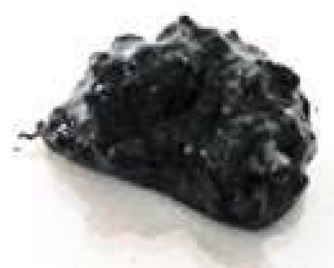

(r)

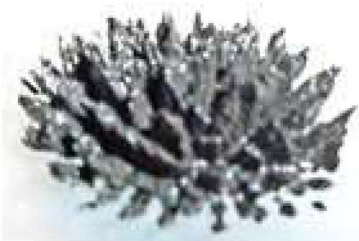

(v)

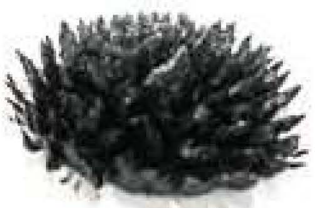

(c)

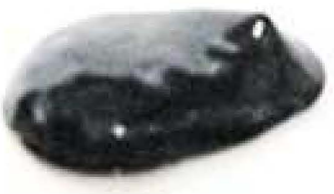

(g)

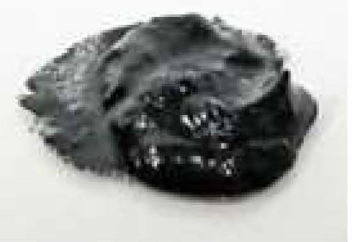

(k)

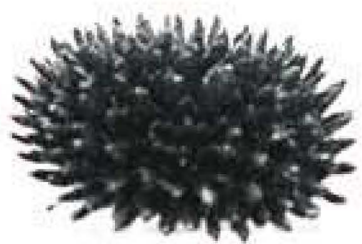

(o)

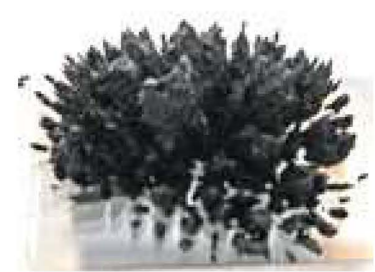

(s)

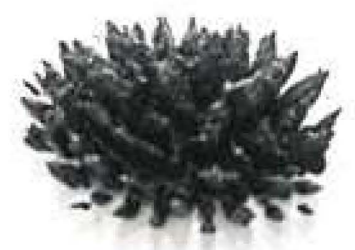

(w)

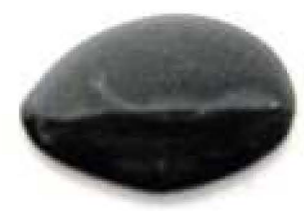

(d)

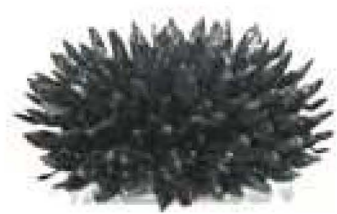

(h)

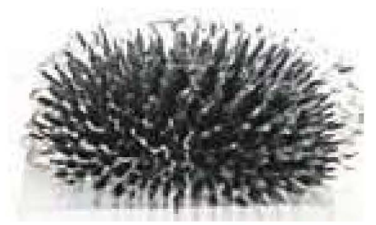

(1)

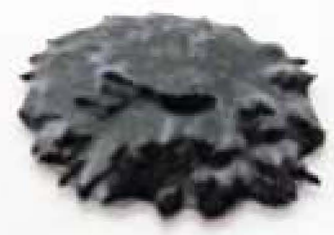

(p)

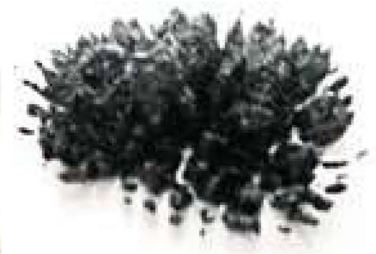

(t)

Figure 1. Photographs of HF specimens (a)-(w), which have the constituents shown in Table 1. 
the same collectability (HF5, HF7, HF8 and HF9). On the other hand, by increasing the PVA or water but not the kerosene or KF96, the sample becomes fluid like water (HF2 and HF4). PVA is so soluble that the fluidity of PVA is close to that of water. The fluid without kerosene is also quite fluid (HF6). Water contributes to fluidity and kerosene contributes to collectability.

When a magnetic field was applied, many spikes were created along the magnetic field line. The spikes are structured by many needle-like magnetic clusters. However, in the specimens with less surfactant or without kerosene (HF3 and HF6), the spikes are not maintained once the magnet is removed. Surfactant and kerosene contribute to the maintenance of the structure of the magnetic clusters.

Regarding magnetic clusters, we can obtain magnetic clusters from HF by using our previously presented technique for the extraction of magnetic clusters from a magnetic responsive fluid such as Magnetorheological Fluid (MRF) or MCF [10]: we put a permanent magnet on the bottom of the beaker into which the fluid is poured, then pour the solvent of the fluid into the beaker and discard it. After many repetitions, we obtain the magnetic clusters on the bottom of the beaker. Figure 2 shows one such HF sample. These magnetic clusters exist in the residual water from the HF solvent. When the magnetic field is removed and the extracted magnetic clusters are shaken, the clusters disperse. However, when the magnetic field is removed without shaking, the configuration of the magnetic clusters is maintained (Figure 2).

The magnetic clusters have a needle-like and dendrite shape and are aligned along the line of the removed magnetic field. The creation of the magnetic clusters induces the enhanced conductivity of the electrolytically polymerized HF rubber.

Given that the combination of water, kerosene, KF96 and PVA brings about collectability as shown in Figure 1, the HF can be guessed to have enhanced viscosity.

The relationship between shear rate and shear stress is shown in Figure 3, which includes HF specimens from both Table 1 and Table 2. W40 in the figure indicates a water-based MF with $40 \mathrm{wt} \% \mathrm{Fe}_{3} \mathrm{O}_{4}$ (W40; Taiho Industry Co., Ltd., Takatsuki, Japan), for comparison with the HF, which replaces MF. For measurement,

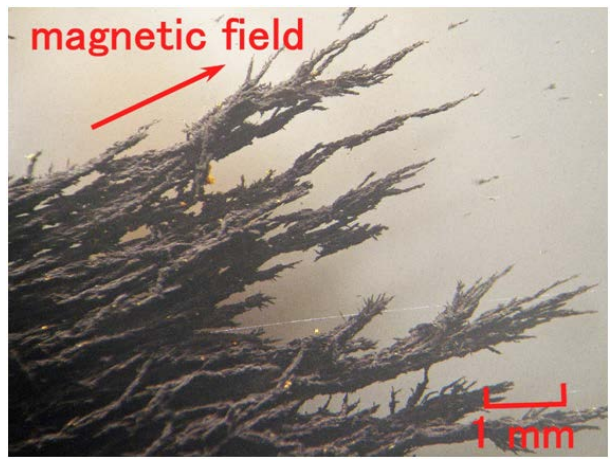

Figure 2. Photograph of magnetic clusters extracted from HF2 aligned along a magnetic field magnified by microscope with magnification of $60 \times$. 


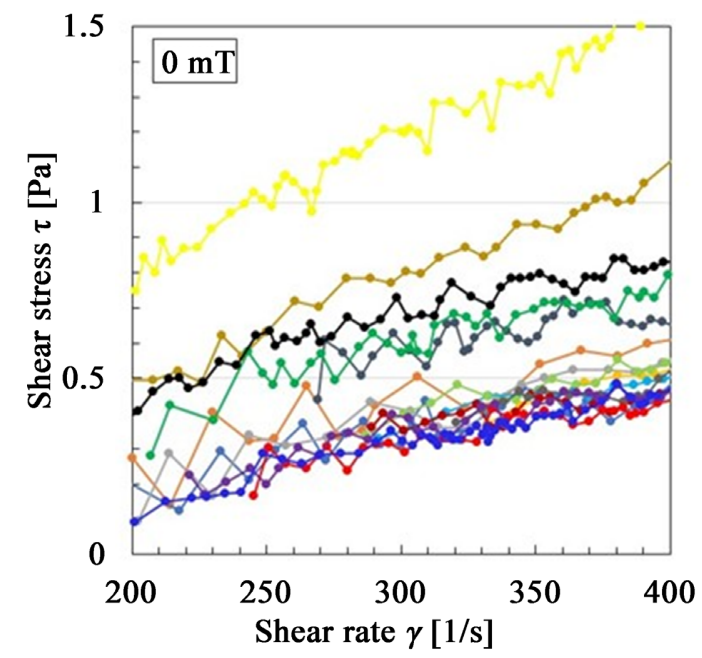

(a)
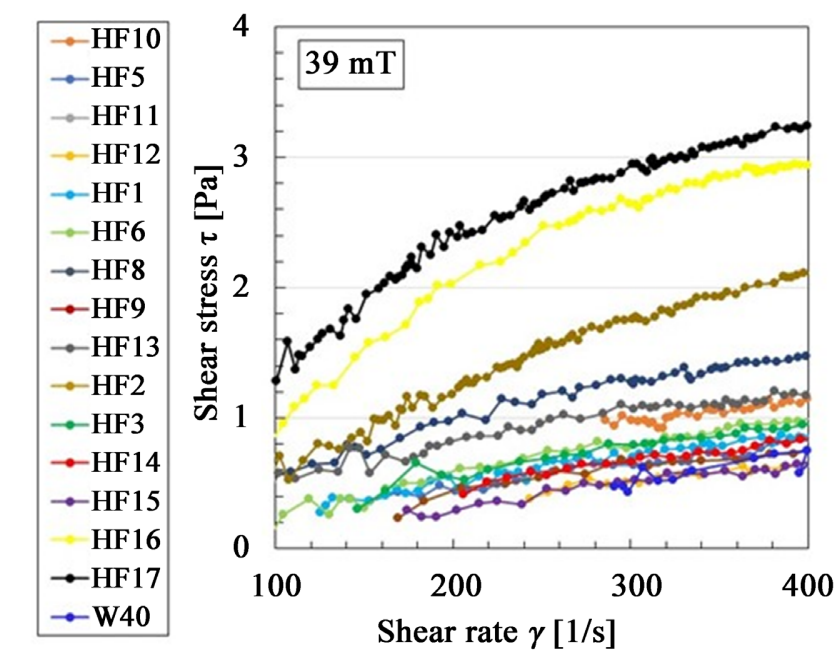

(b)

Figure 3. Relationship between shear rate and shear stress of HFs that have the constituents shown in Table 1 and Table 2: (a) without a magnetic field; (b) under a magnetic field of $39 \mathrm{mT}$.

Table 2. Constituents of other HFs: ${ }^{\star}$, KF96 with $1 \mathrm{cSt}$ replaces one with $100 \mathrm{cSt}$; ${ }^{\star *}$, KF96 with 1 cSt replaces one with $1000 \mathrm{cSt}$ (the viscosity of KF96 of HFs16 and 17 is not different from the one of other HFs).

\begin{tabular}{|c|c|c|c|c|c|c|c|c|}
\hline $\begin{array}{l}\text { Specimen } \\
\text { No. }\end{array}$ & Water $[\mathrm{g}]$ & Kerosene $[\mathrm{g}]$ & KF96 $(1 \mathrm{cSt})[\mathrm{g}]$ & PVA [g] & $\mathrm{Fe}_{3} \mathrm{O}_{4}[\mathrm{~g}]$ & M300 [g] & $\begin{array}{l}\text { Sodium hexadecyl sulfate } \\
\text { aqueous solution }[\mathrm{g}]\end{array}$ & $\begin{array}{l}\text { Density } \\
{\left[\mathrm{kg} / \mathrm{m}^{3}\right]}\end{array}$ \\
\hline HF10 & 3 & 21 & 3 & 3 & 3 & 3 & 4 & 973 \\
\hline HF11 & 21 & 3 & 3 & & 3 & 3 & 4 & 1218 \\
\hline HF12 & 21 & 3 & 3 & 3 & 3 & 3 & 4 & 1181 \\
\hline HF13 & 3 & 3 & 21 & 3 & 3 & 3 & 4 & 991 \\
\hline HF14 & 3 & 3 & 3 & 3 & 1.5 & 1.5 & 4 & 1076 \\
\hline HF15 & 21 & 3 & 3 & 21 & 3 & 3 & 2 & 1098 \\
\hline HF16 & 3 & 3 & $3^{*}$ & 21 & 3 & 3 & 4 & 1091 \\
\hline HF17 & 3 & 3 & $3^{\star *}$ & 21 & 3 & 3 & 4 & 1108 \\
\hline
\end{tabular}

we used a plate-plate type rotational viscometer in order to achieve uniform distribution of the magnetic field over the rotational plate with $40-\mathrm{mm}$ diameter throughout. Torque applied on the plate was measured by torque meter (SS-005, Ono Sokki, Co. Ltd., Yokohama, Japan) under a rotational speed of the plate applied by DC motor (Vexta, BXM460-GFH2, Oriental Motor, Co. Ltd., Tokyo, Japan). The distance between the plates was $1 \mathrm{~mm}$. The magnetic field was applied by a permanent magnet.

When no magnetic field was applied, viscosity increased in HF specimens with KF96 solvent, which has a larger viscosity. Given this result, most HF specimens have the viscosity of MF, which has the same ordered viscosity (water, kerosene, KF96).

Under the application of a magnetic field, the viscosity of HF is multiplied several times, which is the same tendency seen with MF. However, the viscosity 
does not become greater than that of other magnetic responsive fluids, such as MRF. In general, the fluid polymerized by emulsifier has comparatively low viscosity. HF is guessed to have the similar peculiarity.

Figure 4 shows the magnetization of HFs compared to MF measured by a vibrating sample magnetometer (VSM, TM-VSM1015-CRO-T, Tamakawa Co. Ltd., Sendai, Japan). The saturation magnetization of the HF specimens is several times larger than that of MF.

However, it is not larger than that of other magnetic responsive fluids; MRF, for example, is the same regarding viscosity.

HF is coordinate with MF because its viscosity and magnetization are equivalent to those of MF based on the results shown in Figure 3 and Figure 4.

The electrical conductivity of HF2 as a representative of all HFs is shown in Table 3 compared to other liquids. The electrical conductivity of HF is closest to that of PVA, which contains a lot of water. Therefore, HF is inferred to be an oil-in-water $(\mathrm{O} / \mathrm{W})$ emulsion as follows. PVA and surfactant of sodium hexadecyl sulfate are emulsifiers, and emulsions are created by emulsifiers. In general, the dispersed system of emulsion with water phase and kerosene phase is divided in $\mathrm{O} / \mathrm{W}$ emulsion, in which a dispersoid of oil is dispersed in a disperse medium of water, and water-in-oil (W/O) emulsion, in which a dispersoid of water is dispersed in a disperse medium of oil. And liquid phase which can easily dissolve an emulsifier becomes a disperse medium; this is well-known as Bancroft's law. In the present case, the emulsifier is PVA enough to be soluble into water. In addition, the current way to verify whether the dispersal system is $\mathrm{O} / \mathrm{W}$ or $\mathrm{W} / \mathrm{O}$

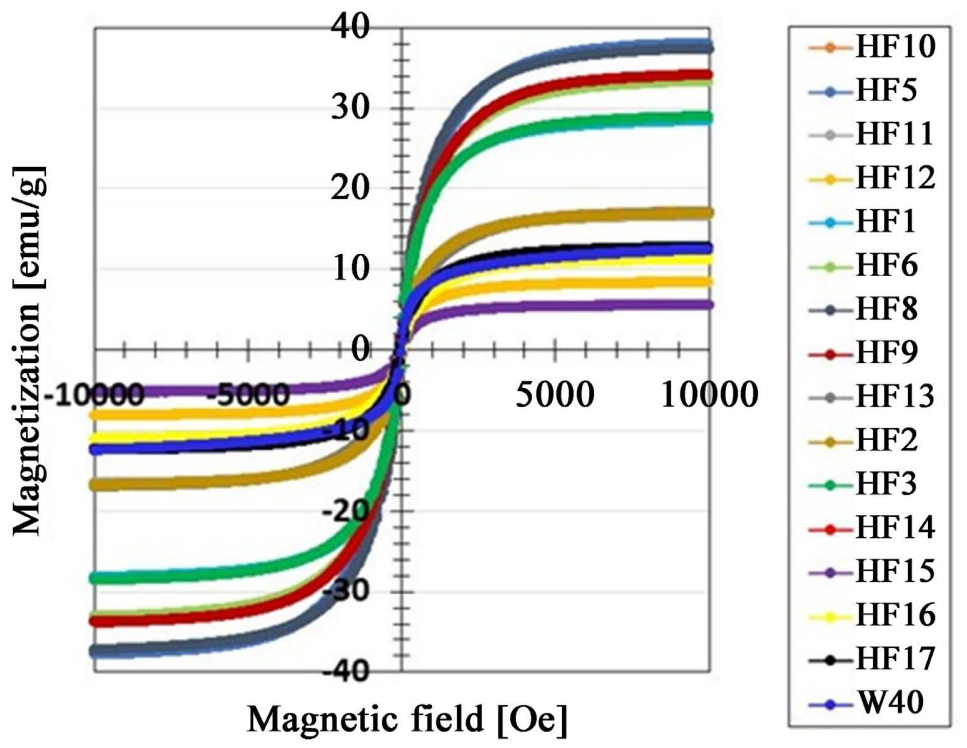

Figure 4. Magnetization of HFs.

Table 3. Electrical conductivity of HF2 compared to the other fluids involved in HF2.

\begin{tabular}{ccccc}
\hline HF & Water & PVA & KF96 (1 cSt) & Kerosene \\
\hline $827 \mu \mathrm{S} / \mathrm{cm}$ & $1.21 \mu \mathrm{S} / \mathrm{cm}$ & $598 \mu \mathrm{S} / \mathrm{cm}$ & $0 \mu \mathrm{S} / \mathrm{cm}$ & $0 \mu \mathrm{S} / \mathrm{cm}$ \\
\hline
\end{tabular}


is based on conductivity: lower conductivity indicates a W/O system, and higher one $\mathrm{O} / \mathrm{W}$.

On the other hand, the viscosity of HF approaches that of water, as seen from the viscosity of HF without a magnetic field (Figure 3(a)). In addition, we can confirm that HF is soluble in water as well as PVA, but not soluble in KF96 or kerosene. Thus, from the above results, we can infer a physical model of HF (Figure 5). As shown in Figure 5(a), PVA is connected between water and KF96 adhered with kerosene. Magnetic particles of $\mathrm{Fe}_{3} \mathrm{O}_{4}$ and $\mathrm{M} 300$ hold the innermost position. Each magnetic particle is coated by PVA, KF96 and kerosene. Thus fabricated scheme does not necessarily connote the overall structure of HF, and the fabricate may be minimized part of HF. Or we may also consider the long-emulsion type (Figure 5(b)), which is easily aggregated by many singleemulsion types such that the PDMS of KF96 has a long-chain molecular structure. In addition to these types, we may also propose other emulsion types, for example, long chain-like aggregated emulsion creations rolling into a ball, a mix of Figure 5(a) and Figure 5(b). As for both types, many emulsion phases agglomerate, creating a large jelly-like body. Therefore, without a magnetic field, HF exhibits a collected body when in a static state, as shown in Figure 6(a). By the application of shear rate, KF96 and kerosene move easily and simultaneously with water and PVA so that HF can flow smoothly, like water. This case may exhibit the deformation of the fabricate of PVA, KF96 and kerosene as shown in Figure 5(b). Therefore, once HF is moved by shear force, it is instantaneously

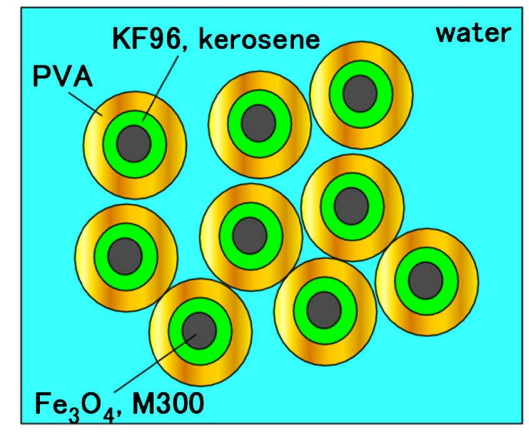

(a)

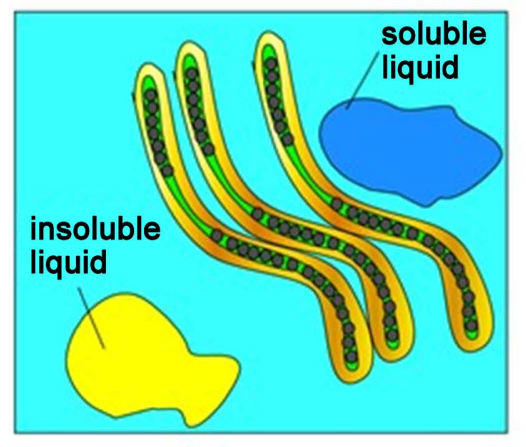

(c)

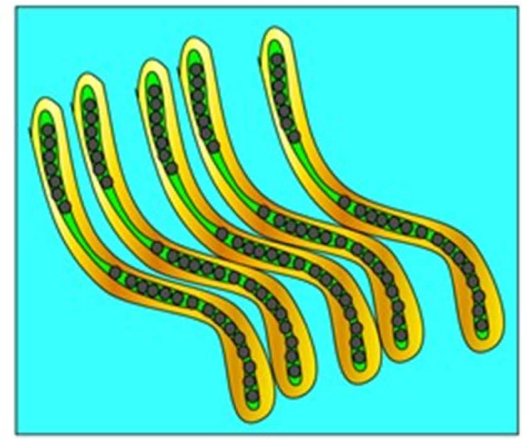

(b)

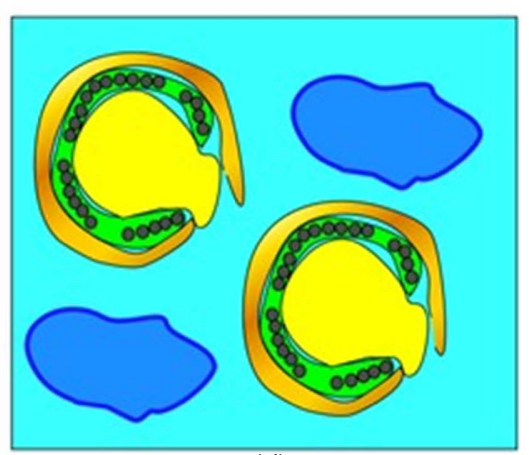

(d)

Figure 5. Physical model of HF: (a) single-emulsion type; (b) long-chain like aggregated emulsion type; (c) and (d) mix of soluble or insoluble liquid with HF. 


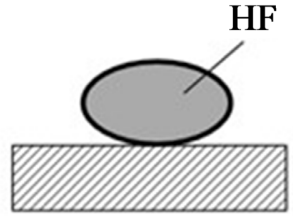

(a)

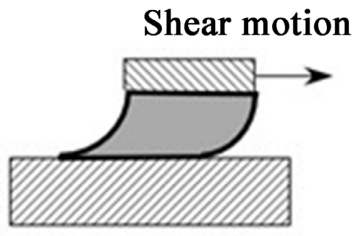

(b)

Figure 6. Schematic shape of the body of the HF: (a) in a static state; (b) under shear motion.

sheared with a fluidic motion, as shown in Figure 6(b).

If soluble or insoluble liquid is mixed with HF as shown in Figure 5(c), the soluble liquid is soluble to water, on the other hand, the insoluble liquid is encompassed by the fabricate of PVA, KF96 and kerosene as shown in Figure 5(d). Therefore HF can be dissolved into whether soluble or insoluble liquid.

\section{Magnetic Conductive Rubber by HF}

When utilizing HF in a rubber, it is important to measure the piezoelectricity and piezoresistivity of the electrolytically polymerized rubber. The HF we used contained $3 \mathrm{~g}$ water, $3 \mathrm{~g}$ kerosene, $3 \mathrm{~g}$ KF96 (1cSt), 3 g PVA, $1.5 \mathrm{~g} \mathrm{Fe}_{3} \mathrm{O}_{4}, 1.5 \mathrm{~g}$ iron particles and $2 \mathrm{~g}$ sodium hexadecyl sulfate aqueous solution. The iron particles used three types of 1.2- $\mu \mathrm{m}$ spherical Fe particles (HQ, Yamaishi Co., Ltd., Noda, Japan), Ni ( $\mu \mathrm{m}$-order and pimple-like shapes on the surface; No. 123; Yamaishi) and M300. HF produced in ad-vance is combined with a rubber latex blended with both $3 \mathrm{~g}$ NR and $3 \mathrm{~g}$ CR (Figures 7(a)-(f)), and a 30-V, 2.7-A electric field is applied to the combined rubber latex under a 312-mT magnetic field for 15 min (Figures $7(\mathrm{~g})-(\mathrm{r})$ ). The electrolytically polymerized HF rubber has a concave-convex surface on the cathode side of the electrode at electrolytic polymerization and a comparatively even surface on the anode side. These are the same characteristics shown by MCF rubber in our previous studies [8] [13], in which the rubber surfaces were different due to the difference between anionic and cationic polymerization [13].

Regarding the HF rubber, we measured the voltage of its piezoelectricity as spontaneous voltage and that of its piezoresistivity as inner voltage obtained by the application of outer voltage, whose mechanisms for generating inner and spontaneous voltages we discuss in our previous study [13], using the same experimental apparatus described in our previous study [13]. Figure 8 and Figure 9 show the results under the repeated and continuous application of a force. In the case of piezoelectricity, the spontaneous voltage using $\mathrm{Ni}$ is larger than those of HQ and M300. Because Ni particles aggregate freely owing to their pimple-like shape, however, spherical particles of HQ and M300 aggregate in a chain-like structure. On the other hand, in the case of piezoresistivity with $10-\mathrm{V}$ outer voltage, the change in voltage with $\mathrm{HQ}$ is larger than that with Ni and M300 because the HQ particle size is small, while that of Ni and M300 is large and the particles aggregate collectively so that electric current flows easily.

From the cause of utilization of the generating spontaneous voltage, we adopt 


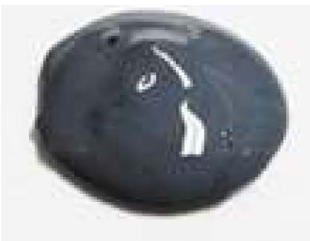

(a)

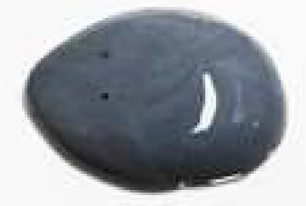

(e)

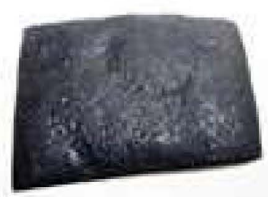

(i)

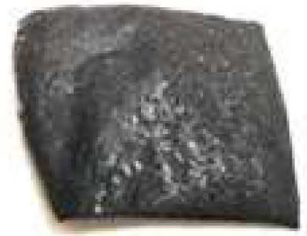

$(\mathrm{m})$

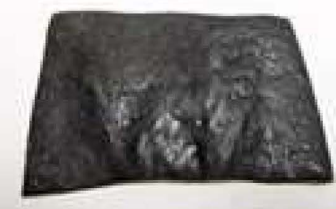

(q)

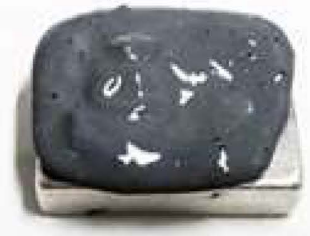

(b)

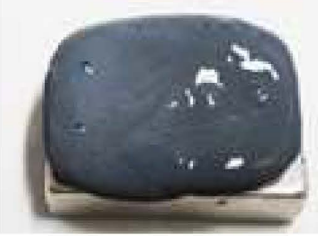

(f)

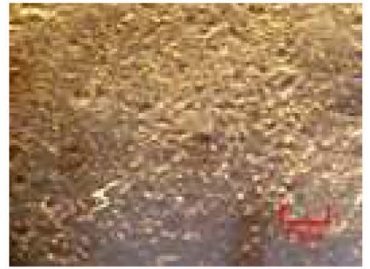

(j)

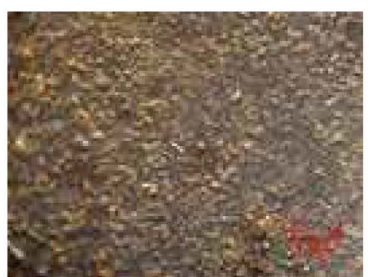

(n)

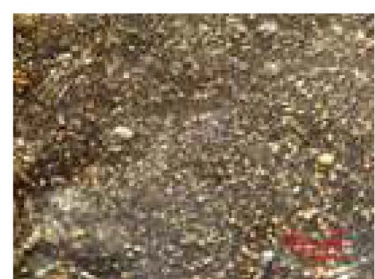

$(\mathrm{r})$

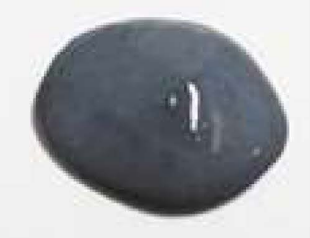

(c)

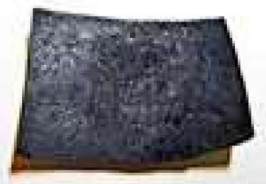

(g)

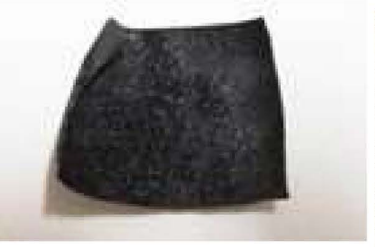

(k)

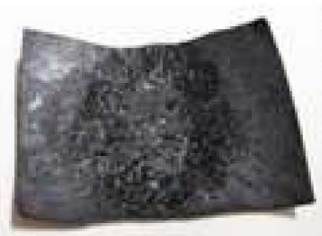

(o)

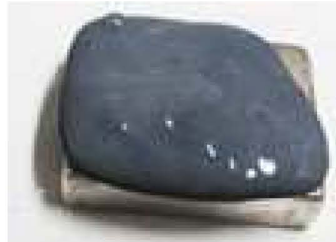

(d)

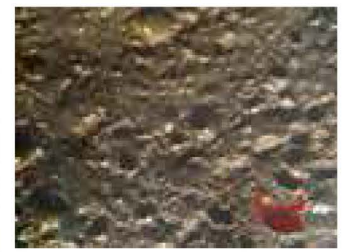

(h)

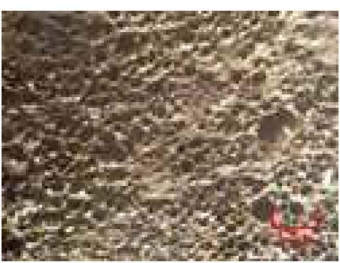

(1)

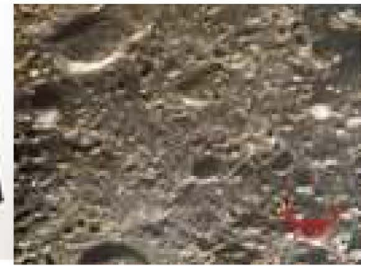

(p)

Figure 7. Photographs of HF combined with rubber (a)-(f) and of electrolytically polymerized HQ rubber (g)-(r): (a), (b), (g)-(j) with HQ; (c), (d), (k)-(n) with Ni; (e), (f), (o)-(r) with M300; (a), (c), (e) without a magnetic field; (b), (d), (f) with the 490-mT magnetic field of a $15 \times 20$-mm permanent magnet; (g), (h), (k), (l), (o), (p) the cathode side of the electrode at electrolytic polymerization; (i), (j), (m), (n), (q), (r) the anode side of the electrode at electrolytic polymerization; (h), (j), (l), (n), (p), (r) magnified by a microscope from (g), (i), (k), (m), (o), (q), respectively.

the utilization of $\mathrm{Ni}$ as optimum filler in the production of HF rubber sensors. It is convenient that the using of an outer power supply connected to a sensor in order to obtain an electrical signal from the sensor by the application of an electric field is dispensable. Because the using of an outer power supply is bulky and heavy, or the using of electric wires is obstructive for operation of robot, the measurement of the generating spontaneous voltage in piezoelectricity is tractable on the operation. 

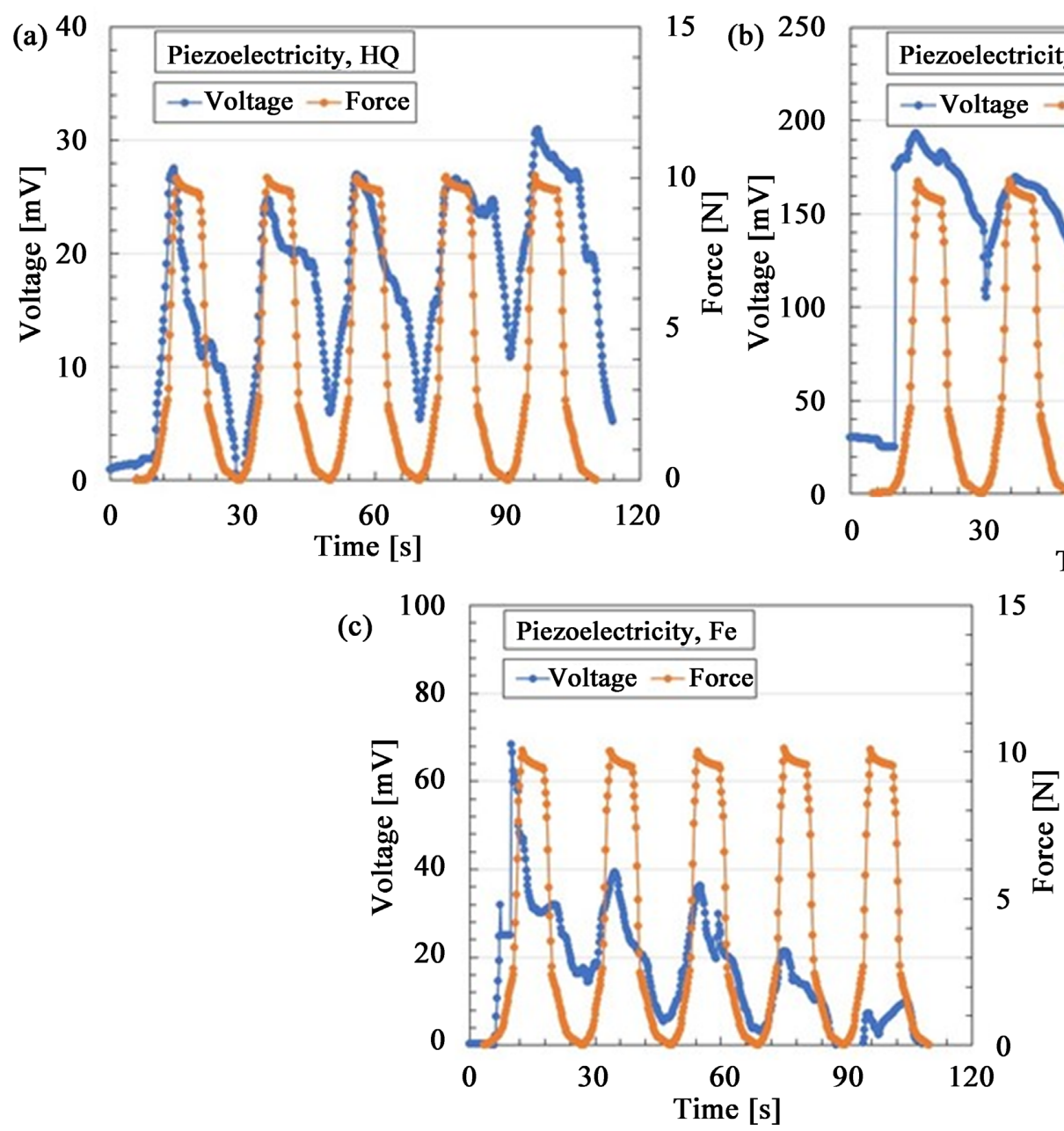

0
15 (b)

250

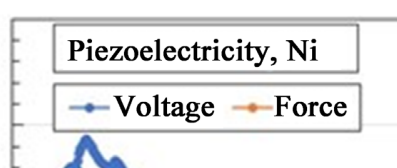

200

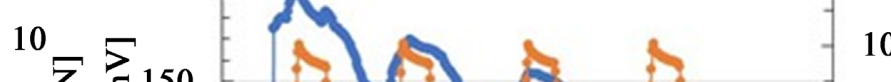

${ }^{1}$

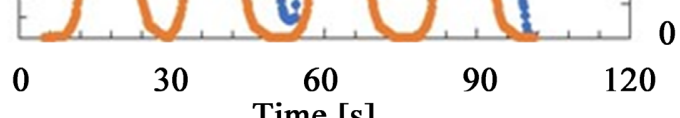

Time $[\mathrm{s}]$

Figure 8. Changes in voltage to force on piezoelectricity in rubber with HF: (a) involving HQ; (b) involving Ni; (c) involving M 300 .

As an example of advancing the effective contribution with HF, by utilizing $\mathrm{HF}$ in the production of tactile rubber, we can present that HF is suitable and easily producible for fabricating rubber with characteristics similar to those of human skin tissue as shown in Appendix [16]. To realize the production of the simulated human skin tissue, the cutaneous receptor in human skin is mimicked to be fabricated by HF rubber, for example, Merkel cells, Meissner corpuscles, etc. By mixing $\mathrm{HF}$ and particles such as $\mathrm{Ni}$ and $\mathrm{TiO}_{2}$ with $\mathrm{NR}, \mathrm{CR}$ and $\mathrm{U}$ as well as by the application of electrolytic polymerization, HF rubber can be fabricated the cutaneous receptors. Furthermore, they are embedded in another human body such as a finger made of $U$ to be molded so that the haptic and stretchable human body with sensibility can be fabricated. HF is effective on the production of their complicated fabrication phases.

\section{Conclusions}

Our novel magnetic responsive fluid, HF, is so useful in the production of conductive soft rubber using the electrolytic polymerization method that it can be 

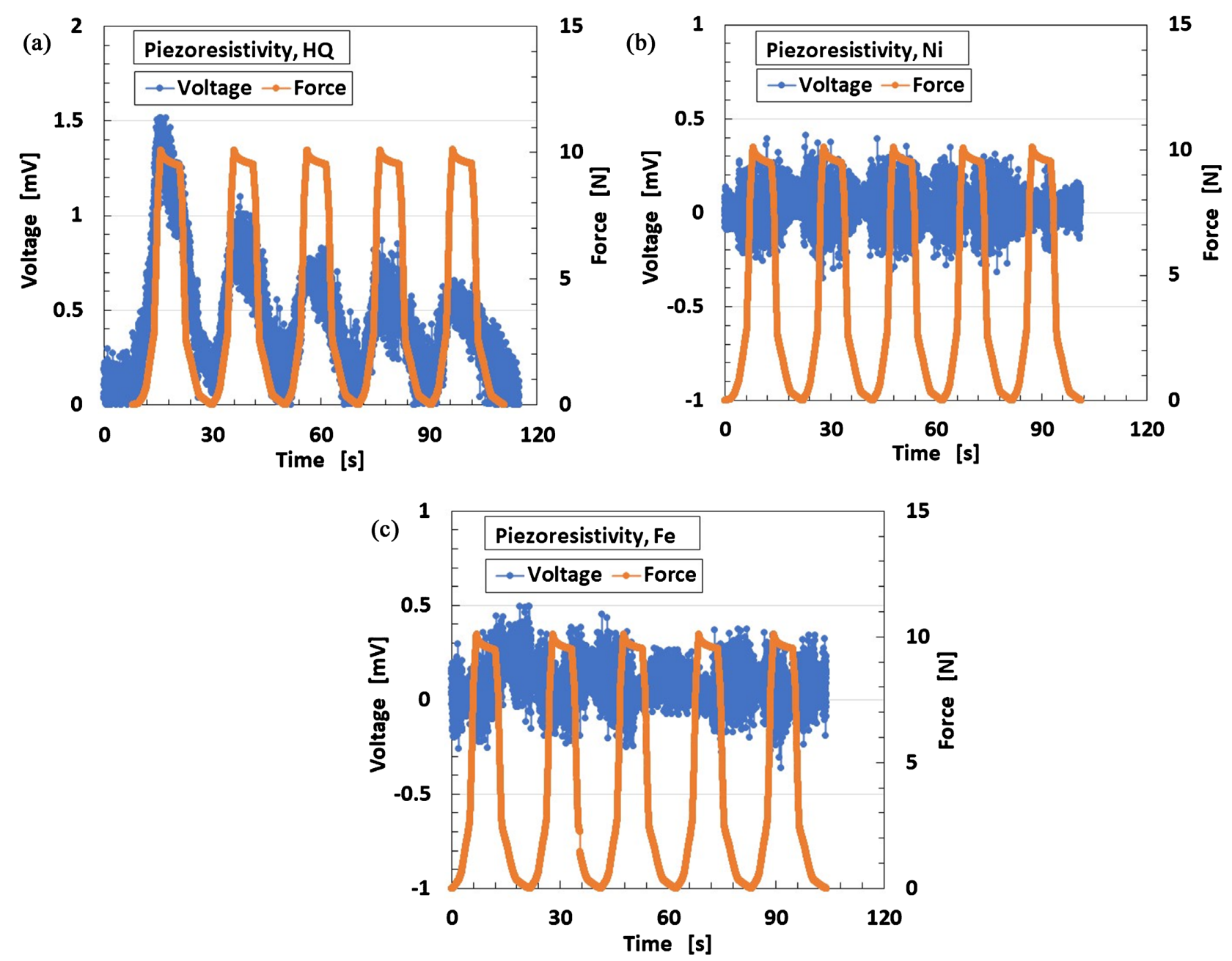

Figure 9. Changes in voltage to force on piezoresistivity in rubber with HF: (a) involving HQ; (b) involving Ni; (c) involving M 300 . 
In another paper, we present the fabrication process of sensitive rubber similar to the sensibility of human skin tissue and the feasibility of replacing MCF with HF. HF is suitable and easily producible for fabricating rubber mimicked a human skin embedded with receptors. Therefore, HF has the viability of soft and tactile rubber like the human skin. Furthermore, because HF is soluble both in water and kerosene or silicon oil, other engineering applications can be expectable. In general, ordinary fluid is categorized soluble to water or soluble to kerosene and silicon oil. Therefore, it is well-known that until recently many engineering applications and scientific phenomena have been developed with taking either soluble or insoluble distinctiveness into account in fluid dynamics. However, the fluid to be soluble simultaneously to water and kerosene, silicon oil is novel that original convention might be created. In addition, because the behavior of HF changes fluidity or jelly-like soft body by the constitution rate of HF, other engineering applications can be also expectable. As is known well, material is divided into three types, solid, fluid and gas. HF institutes the state between solid and fluid such as jelly-like bodies involving the fluid state by controlling the constitution rate of HF. For example, the state of slime or latex, etc. can be easily produced. These states have been utilized in many engineering applications.

\section{Acknowledgments}

This work was supported in part by JSPS KAKENHI Grant Number JP 21K03960 and a grant from the Fukushima Innovation Coast Framework project. We are very grateful for this support.

\section{Conflicts of Interest}

The authors declare no conflicts of interest regarding the publication of this paper.

\section{References}

[1] Liu, P., Liu, C.X., Huang, Y., Wang, W.H., Fang, D., Zhang, Y.G. and Ge, Y.J. (2016) Transfer Function and Working Principle of a Pressure/Temperature Sensor Based on Carbon Black/Silicone Rubber Composites. Journal of Applied Polymer Science, 133, Article ID: 42979. https://doi.org/10.1002/app.42979

[2] Zhang, C.M., Liu, T. and Xu, Y.G. (2015) Piezo-Absorbing Effect of Microwave Absorbing Composites with Carbonyl Iron Particles as the Filler. International Journal of Materials Research, 106, 1086-1093. https://doi.org/10.3139/146.111281

[3] Del Castillo-Castro, T., Casillo-Ortega, M.M., Encinas, J.C., Franco, P.J.H. and Carrillo-Escalante, H.J. (2012) Piezo-Resistance Effect in Composite Based on Cross-Linked Polydimethylsiloxane and Polyaniline: Potential Pressure Sensor Application. Journal of Materials Science, 47, 1794-1802. https://doi.org/10.1007/s10853-011-5965-y

[4] Ogura, K., Ogawa, M., Ohya, K. and Banno, H. (1993) Receiving Characteristics of $\mathrm{d}_{31}$-Zero Piezo-Rubber Hydrophone. Japanese Journal of Applied Physics, 32, 2304 2306. https://doi.org/10.1143/JAP.32.2304

[5] Ruiz, M.M., Marchi, M.C., Perez, O.E., Jorge, G.E., Fascio, M., D’accorso, N. and 
Negri, R.M. (2015) Structured Elastomeric Submillimeter Films Displaying Magneto and Piezo Resistivity. Journal of Polymer Science Part B: Polymer Physics, 53, 574-586. https://doi.org/10.1002/polb.23672

[6] Mahmoud, W.E., El-Lawindy, A.M. Y., El Eraki, M.H. and Hassan, H.H. (2007) Butadiene Acrylonitrile Rubber Loaded Fast Extrusion Furnace Black as a Compressive Strain and Pressure Sensors. Sensors and Actuators A: Physical, 136, 229-233. https://doi.org/10.1016/j.sna.2006.11.017

[7] Pojanavaraphan, T. and Magaraphan, R. (2010) Fabrication and Characterization of New Semiconducting Nanomaterials Composed of Natural Layered Silicates $\left(\mathrm{Na}^{+}-\right.$ MMT), Natural Rubber (NR), and Polypyrrole (PPy). Poly, 51, 1111-1123. https://doi.org/10.1016/j.polymer.2009.07.003

[8] Shimada, K. and Saga, N. (2016) Mechanical Enhancement of Sensitivity in Natural Rubber Using Electrolytic Polymerization Aided by a Magnetic Field and MCF for Application in Haptic Sensors. Sensors, 16, Article No. 1521. https://doi.org/10.3390/s16091521

[9] Shimada, K., Shuchi, S., Kanno, H., Wu, Y. and Kamiyama, S. (2005) Magnetic Cluster and Its Applications. Journal of Magnetism and Magnetic Materials, 289, 9-12. https://doi.org/10.1016/j.jmmm.2004.11.004

[10] Shimada, K., Miyazaki, T., Shibayama, A. and Fujita, T. (2003) Extraction of Magnetic Clusters Self-Assembled by a Magnetic Field. Smart Materials and Structures, 12, 297-303. https://doi.org/10.1088/0964-1726/12/2/318

[11] Shimada, K., Shuchi, S. and Kanno, H. (2005) Magnetic Rubber Having Magnetic Clusters Composed of Metal Particles. Journal of Intelligent Material Systems and Structures, 16, 15-20. https://doi.org/10.1177/1045389X05046687

[12] Shimada, K. (2017) Enhancement of MCF Rubber Utilizing Electric and Magnetic Fields, and Clarification of Electrolytic Polymerization. Sensors, 17, Article No. 767. https://doi.org/10.3390/s17040767

[13] Shimada, K. and Saga, N. (2017) Development of a Hybrid Piezo Natural Rubber Piezoelectricity and Piezoresistivity Sensor with Magnetic Clusters Made by Electric and Magnetic Field Assistance and Filling with Magnetic Compound Fluid. Sensors, 17, Article No. 346. https://doi.org/10.3390/s17020346

[14] Shimada, K., Ikeda, R., Kikura, H. and Takahashi, H. (2019) Development of a Magnetic Compound Fluid Rubber Stability Sensor and a Novel Production Technique via Combination of Natural, Chloroprene and Silicone Rubbers. Sensors, 89, Article No. 3901. https://doi.org/10.3390/s19183901

[15] Shimada, K., Ikeda, R., Kikura, H. and Takahashi, H. (2020) Enhancement of Diversity in Production and Application Utilizing Electro-Lytically Polymerized Rubber Sensors with MCF: 1st Report on Consummate Fabrication Combining Varied Kinds of Constituents with Porous Permeant Stocking-Like Rubber. Sensor, 20, Article No. 4658. https://doi.org/10.3390/s20174658

[16] Shimada, K., Ikeda, R., Kikura, H. and Takahashi, H. (2021) Morphological Fabrication of Rubber Cutaneous Receptors Embedded in a Stretchable Skin Mimicking Human Tissue by the Utilization of Hybrid Fluid. Sensors, 21, Article No. 6834. https://doi.org/10.3390/s21206834 


\section{Appendix}

The originality of the paper to be further analyzed and clarified by the current work is the simulated human skin tissue. The current work is the specific demonstration of the cutaneous receptor embedded in human skin. The cutaneous receptor can be mimicked to be fabricated by HF rubber, for example, Merkel cells, Meissner corpuscles, etc., which has been presented in another paper [16]. Further the fabricated cutaneous receptor is embedded in a human body such as finger so that we can propose a novel artificial human body of skin tissue like a humanoid. Therefore, there is the novelty of HF on this demonstration. Naturally, there would be other novelties except for this demonstration.

We can fabricate with utilizing HF free nerve endings which is shown in Figure A1, Krause and bulbs, Meissner corpuscles, Pacinian corpuscles, Ruffini endings. It is embedded in a finger made of $U$ which is highly stretchable and compressible as shown in Figure A2. Figure A3 shows the voltage of the cutaneous receptors of Krause and bulbs at touching hot or cold water and repeating compression in the water. The voltage is the generated spontaneous voltage in piezoelectricity by using $\mathrm{Ni}$ enough to be useful as shown in Figure 8. The thermal and force responses can be realized such as human finger enough to be expectable for futuristic humanoid.
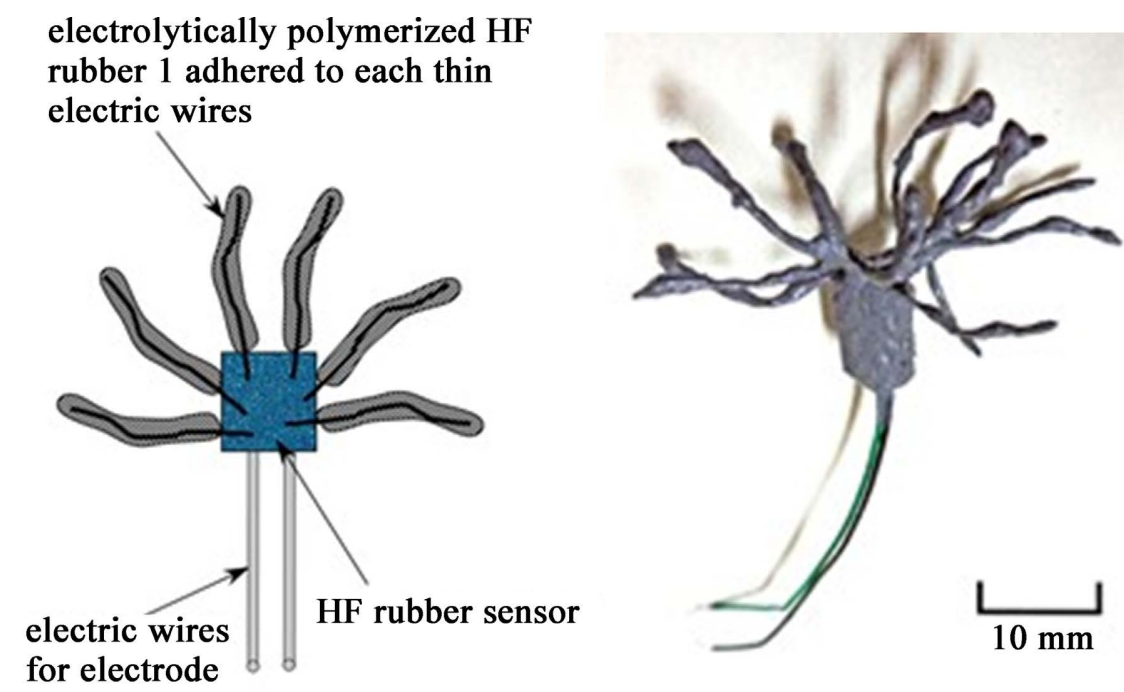

Figure A1. Fabrication of free nerve endings as one of cutaneous receptor as Type-A HF sensor presented in [16]: the left is the schematic diagram; the right is the image.

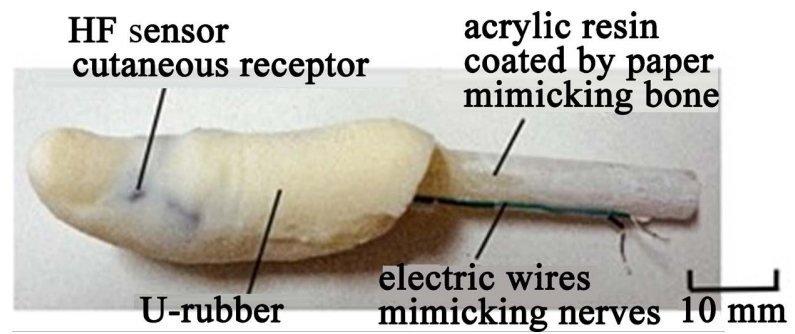

Figure A2. Image of a finger in which the cutaneous receptors are embedded [16]. 


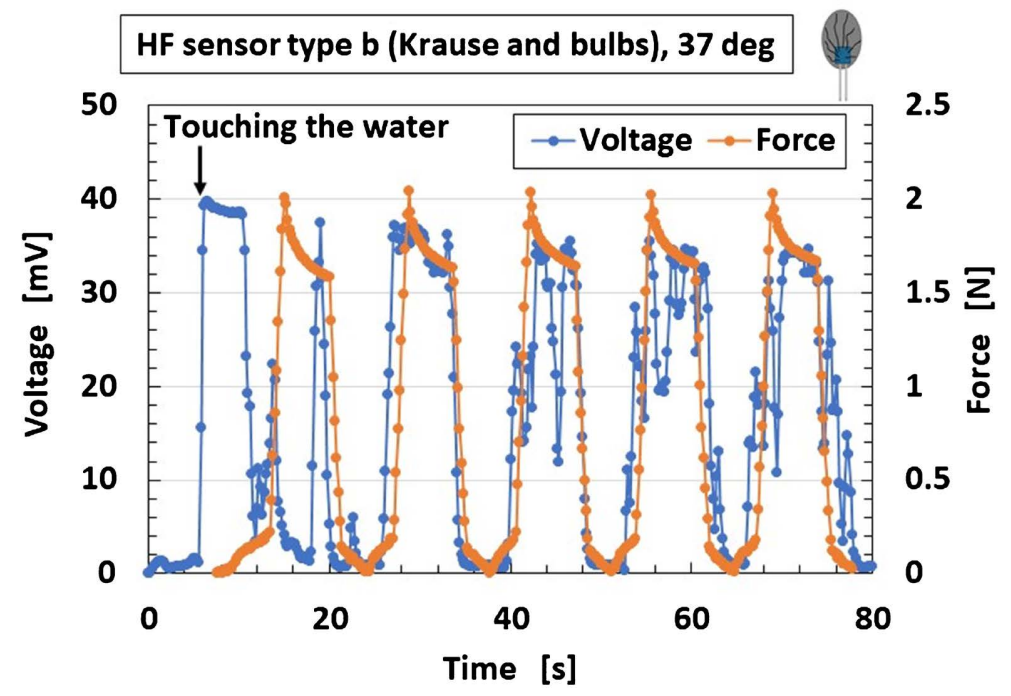

Figure A3. Voltage of the cutaneous receptors of Krause and bulbs at compression [16]. 